\title{
Severe deep neck infections successfully treated with negative pressure wound therapy with instillation - a case report
}

\author{
Krzysztof Szmyt, Adam Bobkiewicz, Łukasz Krokowicz, Tomasz Banasiewicz
}

\section{CASE REPORT}

\begin{abstract}
Background: Deep neck infection (DNI) is a lifethreatening complication associated with significant mortality and morbidity rates. The most common causes of DNI are the tonsilitis, dentitis, salivary glands inflammation, malignancies, and foreign bodies. As a result of neck infection, patients are at high risk of potential secondary complications which include: descending mediastinitis, pleural empyema, septicemia, jugular vein thrombosis, pericarditis. We presented a case of successful management of DNI with the utility of negative pressure wound therapy with instillation (iNPWT).

Method: A 37-year-old male with deep neck infection due to dentitis was qualified for iNPWT. Due to previous incisions and drainage of the neck abscesses, some undermined wounds drained towards each other's were revealed with an excessive amount of purulent content. Standard NPWT dressing was placed and polyurethane foam was covered with contact layer dressing. Additionally, an inflow drain was placed within one of the wounds in regard to instill an antimicrobial solution. The wound was instilled four times daily.

Results: The patient underwent a total of eight iNWPT sessions. Locally, a reduction in purulent content was achieved with a decrease of wounds' dimensions and improvement of wound bed granulation. Moreover, improvement of the patient's general condition and decrease of inflammatory markers was achieved.

Conclusions: iNPWT may play an important role in the management of combined, complicated wounds due to DNI. The instilled antimicrobial solution facilitates dissolving and removing of the purulent content that impairs the wound healing.
\end{abstract}

Keywords-deep neck infection, negative pressure wound therapy, instillation

\section{INTRODUCTION}

D EEP neck infection (DNI) is defined as an infection within potential spaces located in the neck as a result of neck abscess or cellulitis. The most common causes of DNI are tonsilitis, dentitis, salivary glands inflammation, malignancies, and foreign bodies ${ }^{1}$ DNI is a life-threatening complication associated with an estimated mortality rate 0.3 $1.6 \%$ As a result of neck infections, patients are at high risk

Manuscript received 15.04.2020; revised 20.06.2020. This work did not receive any financial support.

Author affiliations: Department of General, Endocrinological Surgery and Gastrointestinal Oncology, Poznan University of Medical Sciences, Poznan, Poland , (KS, AB, KL, TB)

*Correspondence to: Tomasz Banasiewicz: tbnanasiewicz@op.pl of potential secondary complications which include: descending mediastinitis, pleural empyema, septicemia, jugular vein thrombosis, pericarditis ${ }^{3}$ Based on a recent literature review it occurs at a rate of $10-20 \%$ ! 5

The problem of complicated septic wound exists as a result of previous surgical interventions as well as infections, and poses a real challenge in intensive care unit patients. The main goals of wound management are:

1) improving in the patient's general condition,

2) reducing local and systemic septic conditions, and

3) facilitating wound healing.

Holistic management should include extensive debridement, removal of the exudate and purulent discharge, and promotion of tissue granulation.

Current standard wound management in septic wounds is still based on placing drains within the subcutaneous tissue. However, such management is associated with limited effectiveness and does not reduce the severity of local infection.

Introduction of negative pressure wound therapy (NPWT) into the general practice revolutionized the strategy of wound care. ${ }^{6}$ Moreover, the implementation of the instilled antimicrobial solution resulted in significantly better outcomes regarding time to wound healing, reduction in bacterial overload, and advancing hospital discharge ${ }^{7}$ The mechanism of action is based on controlled periodic instillation of a topical solution to the wound bed facilitating removal of cellular debris and cytokines, enhancing excaudate removal and decreasing the bacterial bio-burden.

Application of standard NPWT in wounds complicated with dense purulent discharge, especially those associated with concomitant subcutaneous cavities and pockets may not be sufficient. Moreover, wound exudate has a tendency to agglomerate within natural spaces and impair wound healing. NPWT with instilled antimicrobial solution dissolves and flushes the cellular debris, non-viable tissue, and cytokines. By dissolving the purulent discharge, iNPWT facilitates its removal and penetrates into the wound, disrupting the biofilm 9

We present a case of successful management of deep neck infection with the use of negative pressure wound therapy with instillation (iNPWT). 


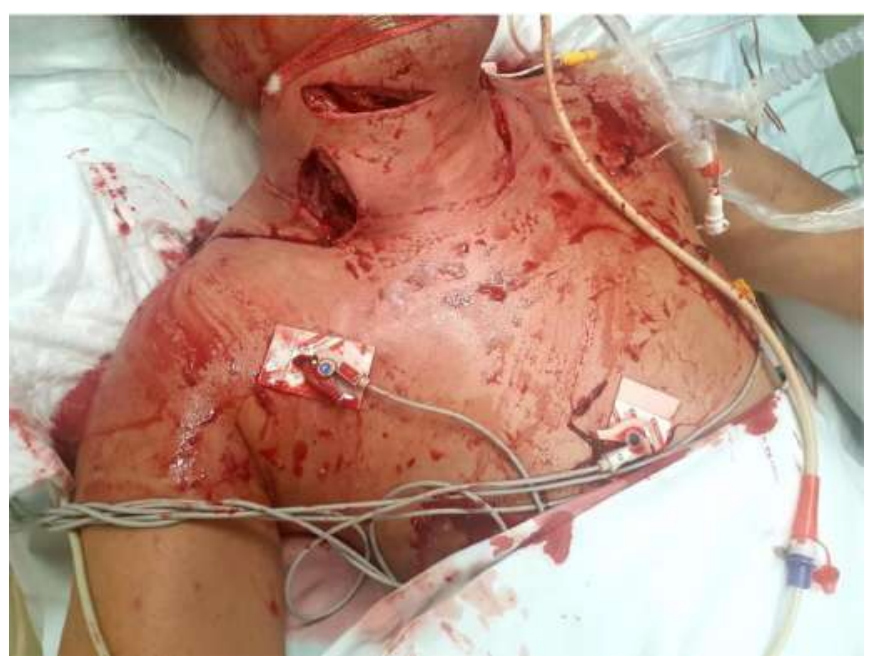

Figure 1. Patient after first surgical intervention - incisions of the neck abscesses.

\section{Case study}

A 37-year-old male was admitted to the intensive care unit due to extensive deep neck infection secondary to odontogenic causes. 3 weeks prior to his admission he had root canal treatment on tooth \#44 and \#47. On the first physical examination, the patient presented with extreme pain, large swelling with severe dyspnea, dysphonia, and dysphagia. Computed tomography (CT) performed in the emergency room showed pus collection on the right side of the neck and right submandibular area.

First, abscesses within the neck had been incised and drained and drains were placed within subcutaneous tissue with the intention of flushing and draining the purulent discharge (Fig. 1) (Fig. 2). Despite the wound's lavage with antimicrobial solution and administration of wide-spectrum antibiotics regimen (piperacillin with tazobactam $4.5 \mathrm{~g} 3$ times per day i.v. and clindamycin $900 \mathrm{mg} 3$ times per day i.v.), there was no improvement in wound healing (Fig. 3). Moreover, a descending inflammation towards the anterior wall of the thoracic cavity was observed. During the next session of dressing change, an additional incision of neck abscess was made and NPWT dressing with instillation was applied. All wounds communicated with each other within the subcutaneous tissue.

Briefly, a standard V.A.C. Dressing System (KCI Medical, San Antonio, USA) was applied. Then, polyurethane (PU) foam was trimmed to an appropriate size. To prevent from ingrowing of granulated tissue within PU foam, a contact layer (Acticoat, Smith \& Nephew Ltd, UK) was used and sutured to the PU foam. After flushing, the undermined cavities and wounds with antimicrobial solution, a superficial debridement of fibrin, and non-viable tissue was made. Then, PU foams covered with the contact layer were precisely applied within the wound bed and secured with stoma paste (Stomahesive paste $\AA$, ConvaTec, USA). Octeniline $\AA$ (Schulke, Warsaw, Poland) was used as an antimicrobial solution.

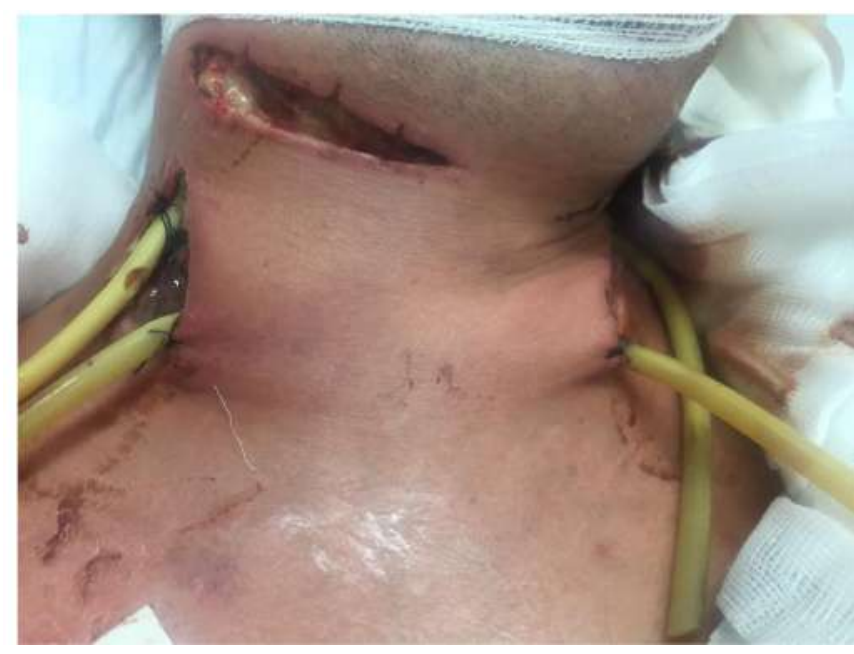

Figure 2. Drains application within the subcutaneous tissue.

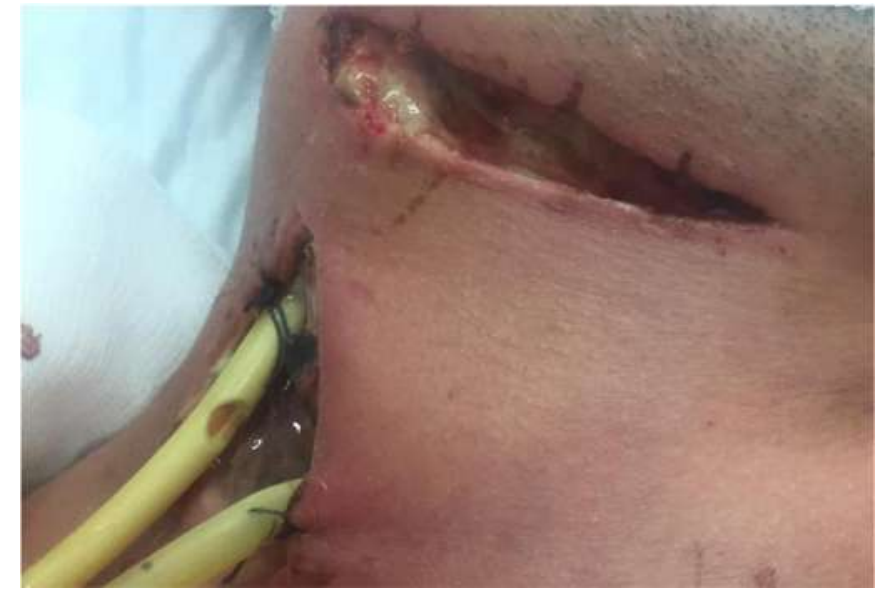

Figure 3. Wounds condition after few days of initial management. No improvement in wound healing.

Additionally, a flexible silicone drain was placed within one of the PU placed on the left side, whereas the port of the iNPWT system was applied within the wound localized in the lowest wound (Fig. 4). Finally, an adhesive drape was placed to keep the system sealed. Such management, applying inflow drain and outflow drain in the lowest wound allowed for distribution and drainage of antimicrobial solution within every undermined part of the wound (Fig. 5). The antimicrobial solution was delivered within NPWT dressing using a $50 \mathrm{ml}$ syringe. Routinely, four times per day an appropriate volume of Octeniline $(R$ was instilled through the drain placed within the wound of the left jugular region. To achieve dwell time, we paused NPWT for 10 minutes. Next, negative pressure was applied again to actively drain the instilled antimicrobial solution. NPWT was set up in the continuous mode with the $-100 \mathrm{mmHg}$ level of negative pressure. The NPWT dressings were changed every three days or on-demand in case of an unsealed system.

After two sessions of iNPWT, we noted an improvement in wound healing: reduction in purulent drainage contents and decreased local signs of the inflammatory response (Fig. 6). 


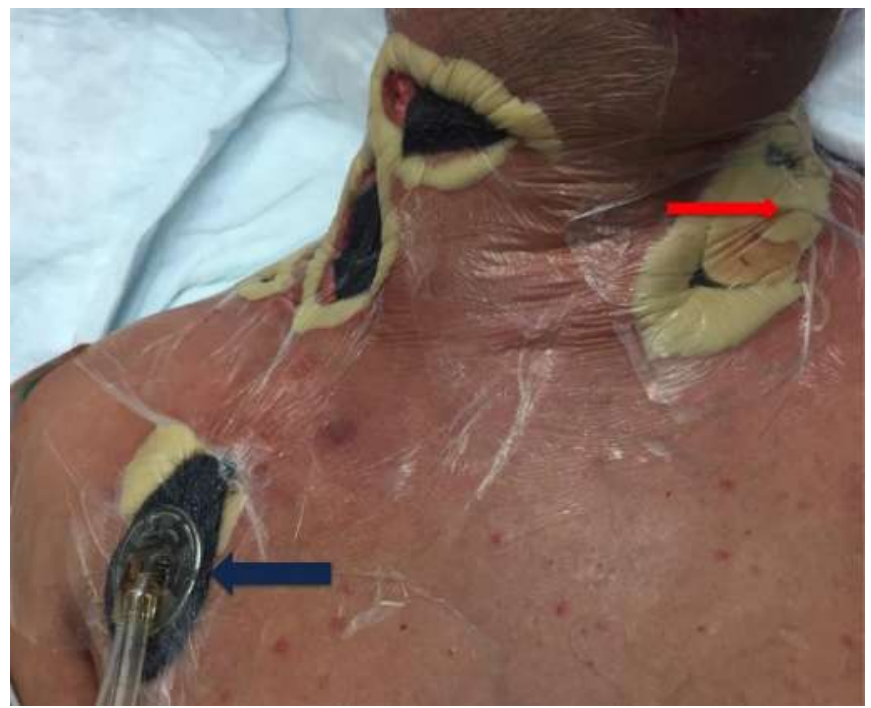

Figure 4. The first application of iNPWT with Octeniline $\mathbb{R}$. Flexible silicone drain $(10 \mathrm{~F})$ was placed inside the incision on the left side (red arrow). NPWT port was localized in the lowest incision (blue arrow).

Simultaneously, the reduction in inflammatory markers: Creactive protein (CRP), procalcitonin (PCT), and white blood cells (WBC) levels were observed.

Every next NPWT dressing change was associated with trimming PU foam to be smaller in the size than the previous PU foam application. Thus, we observed macro-deformation causing a gradual reduction of the wound's size. Because of the proximity of both wounds and some problems with keeping the system sealed, we modified the NPWT strategy. Since PU foam covered with contact layer was placed within the wound bed (as previously described), the skin between wounds was also protected with contact layer and finally, both wounds of neck sites were covered with single PU foam (Fig. 7). For the next four iNPWT sessions, we increased the level of negative pressure to $-125 \mathrm{mmHg}$. After two weeks of iNPWT, an extensive granulation of the wound bed without any purulent content was observed (Fig. 8). We achieved partial secondary closure. Constant improvement in clinical status was obtained and confirmed with imaging studies.

The patient was transferred to the otorhinolaryngology department. A total of eight sessions were performed. We did not observe any complications during the therapy.

\section{Discussion}

DNI is a serious bacterial infection in the presence of neck abscesses, cellulitis or/and phlegmons (from the skull base to the mediastinum). $\frac{10}{n}$ The most common etiology is odontogenic (approximately of $40 \%$ ) — as it was in the presented study. Other common causes include salivary gland infections, head and neck trauma, iatrogenic infections after surgical or dental procedures, neoplasm (benign and malignant) $[5$

The surgical method of choice are incision and drainage of the abscess. Moreover, patients diagnosed with DNIs require advanced dental and respiratory tract procedures and the introduction of a wide spectrum antimicrobial regimen. The

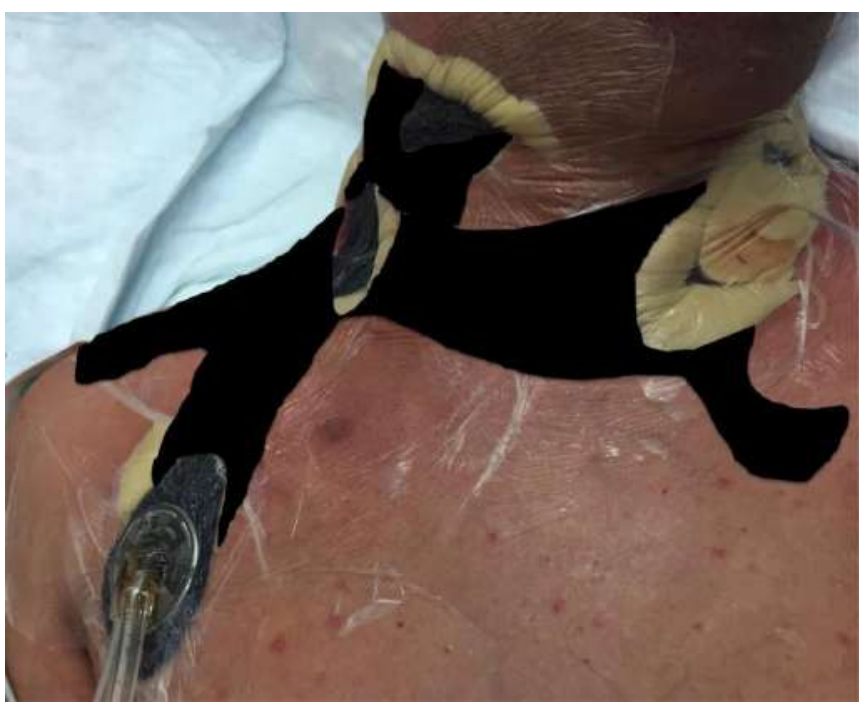

Figure 5. Modified iNPWT allowed for distribution and drainage of antimicrobial solution within entire undermined wound (subcutaneous communication between wounds is marked in black).

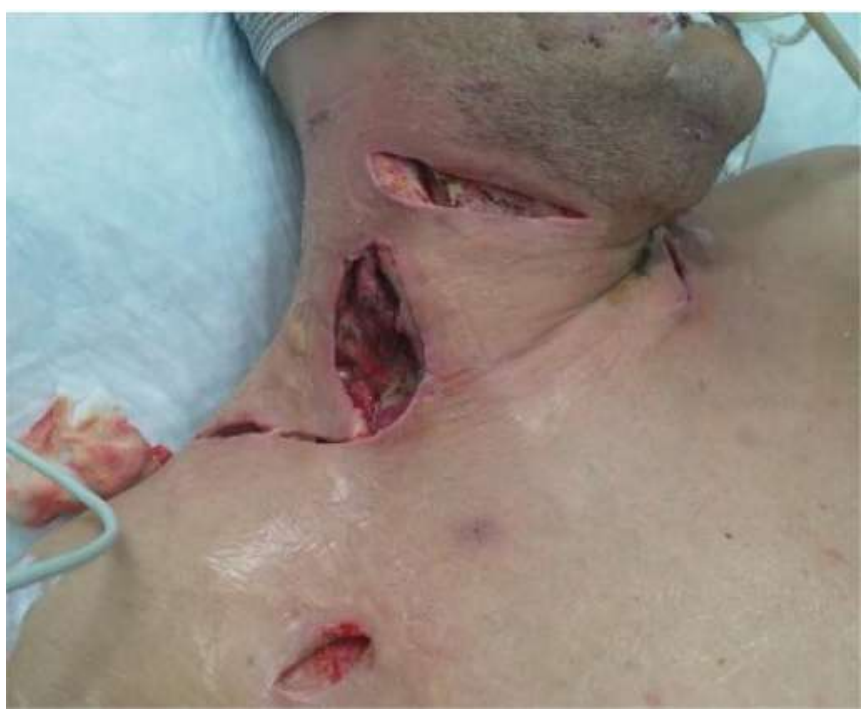

Figure 6. Application of the third iNPWT dressing. Improvement in wound healing, reduction in purulent drainage contents and decreased inflammatory response were observed.

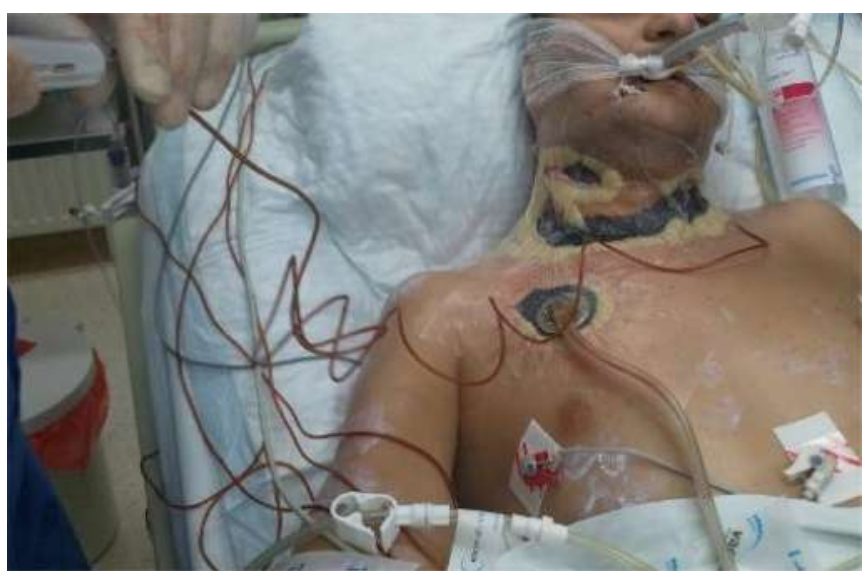

Figure 7. The sixth session of iNPWT. Wounds on both sites of the neck were covered with single piece of PU foam. 


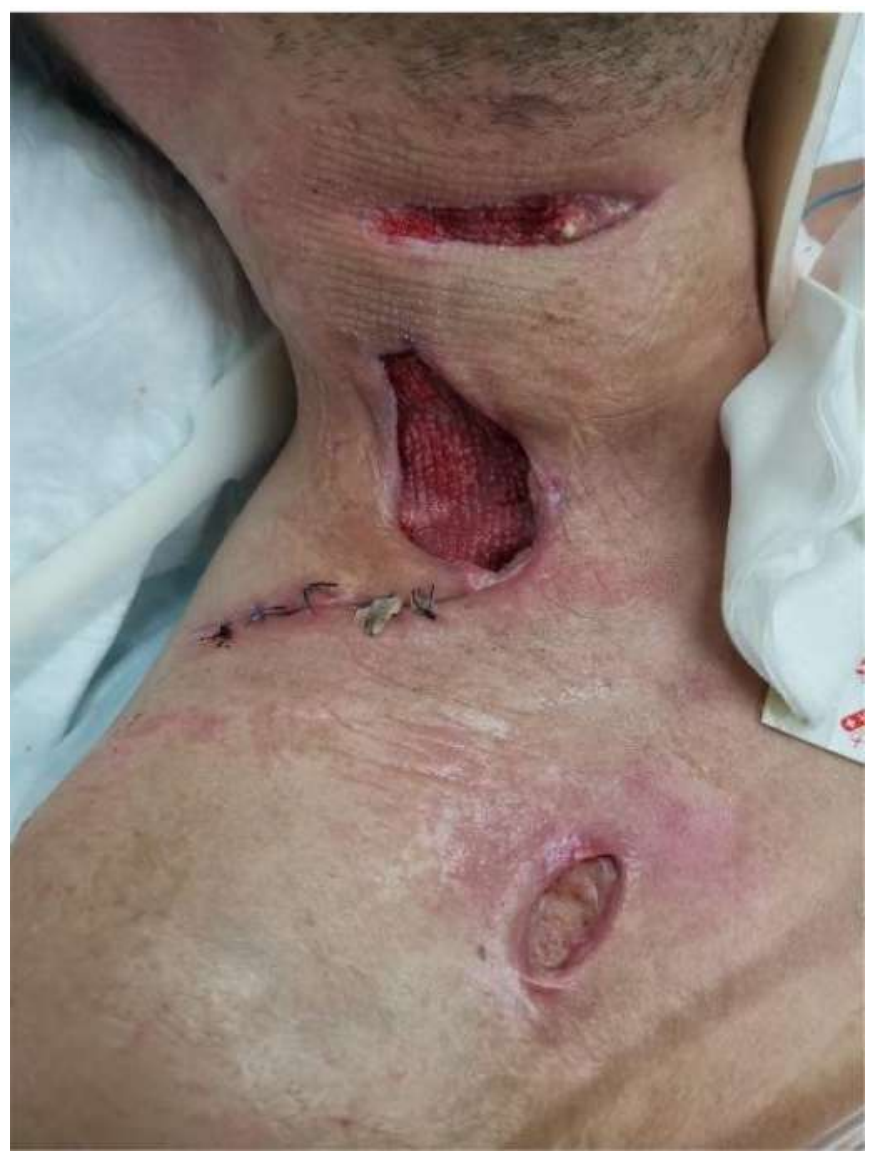

Figure 8. Third week of iNPWT. An extensive granulation of the wound bed with no purulent content was observed

crucial issue is to identify and treat the most underlying pathology responsible for the infection (for example: removal of the infected teeth or tonsil). On the other hand, DNIs may lead to some additional complications and appropriate management of these are crucial. 11 This 'gold' standard treatment, in this case, was ineffective. We introduced iNPWT as a method of choice. Based on our experience, application iNWPT in complicated wounds may serve as an important alternative to standard management. Moreover, iNPWT is an accepted method improving the effectiveness of the standard NPWT therapy and can be helpful in complicated clinical situations, such as the open abdomen (OA) management ${ }^{12}$ or in the treatment of infected implants. ${ }^{13}$ iNPWT can be successfully used in the oral and mixillofacial surgery. 14

In this case study, few clinical challenges were presented. Firstly, it was a serious and severe infection. Secondly, there was a large amount of purulent discharge and no improvement was achieved due to standard management. Thirdly, the localization of the infection within the neck, very close to the fragile, large blood vessels (carotid arteries - especially in the left side, and subclavicular vessels especially on the right side, as well as lungs and trachea). To overcome the mentioned problems, a modified iNPWT was used.

The decision about the type of instilled fluid seems to be crucial. According to recent recommendations, there was no firm conclusion to indicate one standard antimicrobial solution used for iNPWT with a wide range of antiseptic agents. In a presented clinical scenario, Octenilin $\AA$ (octenidinebased wound irrigation solution) was used. Octenilin $($ was created for cleansing and moisturizing chronic wounds and burns. ${ }^{15}$ This system can also be used to loosen encrusted dressings. ${ }^{16}$ Octenidine-based solutions remove necrotic tissue, slough, and debris from the wound bed, and is particularly suitable for difficult-to-access locations, such as fissures and wound pockets ${ }^{17}$ The important benefit of iNPWT with Octenilin $($ ) was the effective evacuation of thick purulent discharge. This method prevents biofilm formation and helps in biofilm defragmentation. Cutting et al. demonstrated that

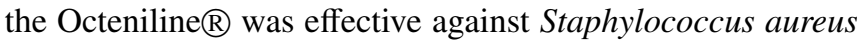
biofilms. In that paper, authors showed almost complete removal of a biofilm 24-hour after starting the therapy with Octenilin $₫ !$

Another clinical problem was the localization of the wounds with the occurrence of fragile structures located close to them. Direct contact of PU sponge with exposed blood vessels is contraindicated because of the potential risk of vessel laceration and bleeding during the therapy and during dressing changes.

To avoid these complications, the authors recommended a contact layer - special non-adhesive fenestrated wound dressing added between the PU sponge and wound bed. That method was safe and widely accepted as a modification of the standard NPWT treatment ${ }^{19}$ Application of PU foam in the undermined wounds facilitates: 1) to keep the system sealed and 2) the adequate soaked of Octeniline $(\mathbb{R}$ through the wound.

On the other hand, different dressings may be used in such a clinical situation. ${ }^{20}$ Another option, dedicated for this type of wound is polyvinyl alcohol foam (PVA). Unfortunately, in the presented case the purulent exudation was too dense to be effectively suctioned by PVA foam.

To avoid any potential complication with bleeding, -100 $\mathrm{mmHg}$ as a level of negative pressure was used during iNPWT sessions. In the authors' opinion, that range of pressure is safe for fragile structures such as blood vessels or nerves. After the decrease of the local inflammation and improvement of granulated tissue, an increased level of negative pressure $(-125 \mathrm{mmHg})$ was used.

\section{Conclusions}

In our opinion, iNPWT may play an important role in the management of combined, complicated wounds due to DNI. The subcutaneous application of NPWT was a promising and important technical trick and tip. The utility of iNPWT improves drainage, decreases inflammation, and protect the surrounding skin from irritation. Installed antimicrobial

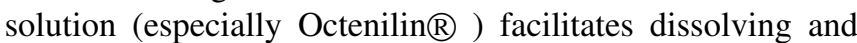
removing of the purulent content that impairs wound healing.

Modification of iNPWT as presented in the study, seems to be a crucial element for good outcomes. 


\section{REFERENCES}

[1] T.-Y. Wong, "A nationwide survey of deaths from oral and maxillofacial infections: the taiwanese experience," Journal of oral and maxillofacial surgery, vol. 57, no. 11, pp. 1297-1299, 1999.

[2] D. Dalla Torre, S. Brunold, I. Kisielewsky, F. R. Kloss, and D. Burtscher, "Life-threatening complications of deep neck space infections," Wiener klinische Wochenschrift, vol. 125, no. 21-22, pp. 680-686, 2013.

[3] F. Vieira, S. M. Allen, R. M. S. Stocks, and J. W. Thompson, "Deep neck infection," Otolaryngologic Clinics of North America, vol. 41, no. 3, pp. 459-483, 2008.

[4] S. Bakir, M. H. Tanriverdi, R. Gün, A. E. Yorgancilar, M. Yildirim, G. Tekbaş, Y. Palanci, K. Meriç, and İ. Topçu, "Deep neck space infections: a retrospective review of 173 cases," American journal of otolaryngology, vol. 33, no. 1, pp. 56-63, 2012.

[5] P. Boscolo-Rizzo, M. Stellin, E. Muzzi, M. Mantovani, R. Fuson, V. Lupato, F. Trabalzini, and M. C. Da Mosto, "Deep neck infections: a study of 365 cases highlighting recommendations for management and treatment," European Archives of Oto-Rhino-Laryngology, vol. 269, no. 4, pp. 1241-1249, 2012.

[6] W. Fleischmann, W. Strecker, M. Bombelli, and L. Kinzl, "Vacuum sealing as treatment of soft tissue damage in open fractures," Der Unfallchirurg, vol. 96, no. 9, pp. 488-492, 1993.

[7] A. Gabriel, J. Shores, C. Heinrich, W. Baqai, S. Kalina, N. Sogioka, and S. Gupta, "Negative pressure wound therapy with instillation: a pilot study describing a new method for treating infected wounds," International wound journal, vol. 5, no. 3, pp. 399-413, 2008.

[8] P. J. Kim, C. E. Attinger, O. Olawoye, B. D. Crist, A. Gabriel, R. D. Galiano, S. Gupta, J. Lantis Ii, L. Lavery, B. A. Lipsky et al., "Negative pressure wound therapy with instillation: review of evidence and recommendations," Wounds, vol. 27, no. 12, pp. S2-S19, 2015.

[9] A. Bobkiewicz, A. Studniarek, M. Drews, and T. Banasiewicz, "Negative pressure wound therapy with instillation (npwti): Current status, recommendations and perspectives in the context of modern wound therapy." Negative Pressure Wound Therapy Journal, vol. 3, no. 1, 2016.

[10] T. M. Osborn, L. A. Assael, and R. B. Bell, "Deep space neck infection: principles of surgical management," Oral and maxillofacial surgery clinics of North America, vol. 20, no. 3, pp. 353-365, 2008.

[11] L.-F. Wang, W.-R. Kuo, S.-M. Tsai, and K.-J. Huang, "Characterizations of life-threatening deep cervical space infections: a review of one hundred ninety-six cases," American journal of otolaryngology, vol. 24, no. 2, pp. 111-117, 2003.

[12] M. R. Matthews, A. N. Quan, A. S. Weir, K. N. Foster, and D. M Caruso, "Temporary abdominal closure combined with an irrigating system utilizing hypochlorous acid solution to decrease abdominal mucopurulence," Eplasty, vol. 18, 2018.

[13] J. D. Hehr, T. S. Hodson, J. M. West, S. A. Schulz, S. J. Poteet, R. Y. Chandawarkar, and I. L. Valerio, "Instillation negative pressure wound therapy: An effective approach for hardware salvage," International Wound Journal, vol. 17, no. 2, pp. 387-393, 2020.

[14] F. M. Eckstein, V. Pinsel, M. C. Wurm, A. Wilkerling, E.-M. Dietrich, S. Kreißel, C. von WIlmowsky, and T. Schlittenbauer, "Antiseptic negative pressure instillation therapy for the treatment of septic wound healing deficits in oral and maxillofacial surgery," Journal of CranioMaxillofacial Surgery, vol. 47, no. 3, pp. 389-393, 2019.

[15] I. B. de Mattos, S. P. Nischwitz, A.-C. Tuca, F. Groeber-Becker, M. Funk, T. Birngruber, S. I. Mautner, L.-P. Kamolz, and J. C. Holzer, "Delivery of antiseptic solutions by a bacterial cellulose wound dressing: Uptake, release and antibacterial efficacy of octenidine and povidone-iodine," Burns, 2019.

[16] J. Matiasek, P. Kienzl, L. W. Unger, C. Grill, R. Koller, and B. R. Turk, "An intra-individual surgical wound comparison shows that octenidinebased hydrogel wound dressing ameliorates scar appearance following abdominoplasty," International wound journal, vol. 15, no. 6, pp. 914 920, 2018.

[17] P. CHADWICK, N. IVINS, M. PILCHER, and J. STEPHENHAYNES, "Case studies: Octenilin wound irrigation solution and octenilin wound gel in practice." Wounds UK, 2016.

[18] K. F. Cutting and S. J. Westgate, "The use of wound cleansing solutions in chronic wounds." Wounds UK, vol. 8, no. 4, 2012.

[19] T. Banasiewicz, B. Banky, A. Karsenti, J. Sancho, J. Sekáč, and D. Walczak, "Traditional and single use npwt: when to use and how to decide on the appropriate use? recommendations of an expert panel." Wounds International., vol. 10, no. 3, pp. 56-62, 2019.

[20] D. DOMAGALSKA, A. BOBKIEWICZ, T. DREWA, and T. BANASIEWICZ, "Urgotul $($ ag/silver dressing as an intermediate layer in negative pressure wound therapy in a patient with a chronic wound and history of multiple laparotomies." Leczenie Ran, vol. 15, no. 2, 2018. 